\title{
A Retrospective Evaluation of Chemotherapy Regimens in Unselected Patients with Metastatic Non-Small Cell Lung Cancer
}

\author{
Ahmed Ashour Badawy ${ }^{1 *}$, Abbas Omar ${ }^{1}$, Waleed Arafat ${ }^{1}$, Gehan Khedr ${ }^{1}$, Sejong Bae ${ }^{2}$, \\ Stefan Grant ${ }^{3}$ \\ ${ }^{1}$ Clinical Oncology and Nuclear Medicine Department, University of Alexandria Faculty of Medicine, Alexandria, Egypt \\ ${ }^{2}$ Preventive Medicine Division, University of Alabama at Birmingham, Birmingham, AL, USA \\ ${ }^{3}$ Hematology oncology Division, Wake Forest University Comprehensive Cancer Center, Winston Salem, NC, USA \\ Email:*asure_egypt@yahoo.com
}

How to cite this paper: Badawy, A.A., Omar, A., Arafat, W., Khedr, G., Bae, S. and Grant, S. (2018) A Retrospective Evaluation of Chemotherapy Regimens in Unselected Patients with Metastatic Non-Small Cell Lung Cancer. Journal of Cancer Therapy, 9 , 314-322.

https://doi.org/10.4236/jct.2018.93029

Received: February 5, 2018

Accepted: March 25, 2018

Published: March 28, 2018

Copyright $\odot 2018$ by authors and Scientific Research Publishing Inc. This work is licensed under the Creative Commons Attribution International License (CC BY 4.0).

http://creativecommons.org/licenses/by/4.0/

(c) (i) Open Access

\begin{abstract}
Background: Randomized clinical trials have demonstrated the benefits of chemotherapy in carefully selected non-small cell lung cancer (NSCLC) patients. How generalizable these results are to other NSCLC patients is unresolved. Methods: The outcomes of patients treated with standard chemotherapy regimens (paclitaxel/carboplatin; gemcitabine/carboplatin; pemetrexed/carboplatin; paclitaxel/carboplatin/bevacizumab) off study as first line therapy between 2002 and 2012 at our institution were compared to the reported results of trials supporting the FDA approval of these drugs and/or regimens. Results: In our population, $38.1 \%$ of the patients had hypertension, $11.9 \%$ of the patients were diabetic, $23.7 \%$ had chronic obstructive pulmonary disease (COPD), $11.9 \%$ had coronary artery disease (CAD) and $2.1 \%$ had renal or liver disease. Notably, the presence of a single or multiple comorbidities was associated with low overall survival compared to matched patients with no comorbidities $(p=0.007)$. Conclusion: The presence of single or multiple comorbidities is associated with inferior overall survival compared to matched groups without such pre-existing conditions.
\end{abstract}

\section{Keywords}

NSCLC-Lung Cancer Chemotherapy-Comorbidities

\section{Introduction}

Advanced stage lung cancer is a lethal disease with an estimated 1.59 million deaths annually worldwide, lung cancer accounting for $19.4 \%$ of total cancer 
death [1] [2].

Lung cancer is divided into two main categories non-small cell lung cancer (NSCLC), which accounts for approximately $85 \%$ of cases and small cell lung cancer [3] [4].

Historically, the median survival of untreated patients with stage IV NSCLC was only 4 - 5 months [5]. The introduction of effective chemotherapy resulted in marked improvements in the survival of metastatic NSCLC patients. The benefit of chemotherapy in metastatic NSCLC was demonstrated in a meta-analysis conducted by the Non-Small Cell Lung Cancer Collaborative Group that included 16 randomized controlled trials in which 2714 patients were randomized to chemotherapy vs. best supportive care. Results showed a significant benefit of chemotherapy (HR, $0.77 ; 95 \% \mathrm{CI}, 0.71$ to $0.83 ; \mathrm{P}=0.0001$ ), equivalent to a relative increase in survival of $23 \%$ or an absolute improvement in survival of $9 \%$ at 12 months, increasing survival from $20 \%$ to $29 \%$ at one year [6].

Since then, many randomized trials were conducted to identify best regimens in metastatic NSCLC. The Eastern Cooperative Oncology Group (ECOG) conducted a randomized trial comparing the efficacy of four commonly used regimens. Patients with advanced NSCLC were randomly assigned to receive: cisplatin $75 \mathrm{mg} / \mathrm{m}^{2}$ and paclitaxel $75 \mathrm{mg} / \mathrm{m}^{2}$ every 3 weeks; cisplatin $100 \mathrm{mg} / \mathrm{m}^{2}$ on day 1 and gemcitabine $1000 \mathrm{mg} / \mathrm{m}^{2}$ on days 1,8 , and 15 every 4 weeks; cisplatin 75 $\mathrm{mg} / \mathrm{m}^{2}$ and docetaxel $75 \mathrm{mg} / \mathrm{m}^{2}$ every 3 weeks; or, carboplatin area under the curve (AUC) of $6 \mathrm{mg} / \mathrm{mL} / \mathrm{min}$ and paclitaxel $225 \mathrm{mg} / \mathrm{m}^{2}$ every 3 weeks. There were no significant differences in response rates, survival, or the time to disease progression between these regimens. The response rate for all regimens was 19 percent, with a median survival of 7.9 months (95\% confidence interval 7.3 - 8.5 months), a 1-year survival rate of 33 percent (95\% CI 30\% - 36\%), and a 2-year survival rate of 11 percent ( $95 \%$ CI, $8 \%$ - 12\%) [7].

The European Organization for Research and Treatment of Cancer (EORTC) compared paclitaxel $175 \mathrm{mg} / \mathrm{m}^{2}$ (day 1 ) or gemcitabine $1250 \mathrm{mg} / \mathrm{m}^{2}$ (days 1 and 8), each combined with cisplatin $80 \mathrm{mg} / \mathrm{m}^{2}$ (day 1) or paclitaxel $175 \mathrm{mg} / \mathrm{m}^{2}$ (day 1) plus gemcitabine $1250 \mathrm{mg} / \mathrm{m}^{2}$ (days 1 and 8) in 480 patients with metastatic NSCLC. There were no statistically significant differences in overall survival between these regimens with median survivals of $8,7.4$ and 6.9 months, respectively. There also was no difference in progression free survival between treatment arms with median progression free survivals of 4.2, 5.1 and 3.5 months, respectively [8].

The Four Arm Cooperative Study (FACS) Group in Japan conducted a randomized clinical trial in which patients with metastatic NSCLC were randomly assigned to one of four regimens: cisplatin $80 \mathrm{mg} / \mathrm{m}^{2}$ on day 1 plus irinotecan 60 $\mathrm{mg} / \mathrm{m}^{2}$ on days $1,8,15$ every 4 weeks; carboplatin AUC $6.0 \mathrm{~min} / \mathrm{mg} / \mathrm{mL}$ on day 1 plus paclitaxel $200 \mathrm{mg} / \mathrm{m}^{2}$ on day 1 every 3 weeks; cisplatin $80 \mathrm{mg} / \mathrm{m}^{2}$ on day 1 plus gemcitabine $1000 \mathrm{mg} / \mathrm{m}^{2}$ on days 1,8 every 3 weeks; or cisplatin $80 \mathrm{mg} / \mathrm{m}^{2}$ on day 1 plus vinorelbine $25 \mathrm{mg} / \mathrm{m}^{2}$ on days 1,8 every 3 weeks. Again, there were no statistically significant differences in response rates or overall survival 
between all these regimens, with all four regimens being well tolerated [9].

The choice between cisplatin or carboplatin remains somewhat controversial. Although randomized clinical trials have shown equivalent overall survival for regimens containing cisplatin or carboplatin, two meta-analyses addressed difference in response rates between the two drugs. Hotta and his colleagues reviewed eight randomized trials that compared cisplatin vs. carboplatin in 2948 patients with metastatic NSCLC. Cisplatin-based chemotherapy produced a higher response rate, but the survival advantage was not shown to be statistically significant (HR1.050; 95\% CI, 0.907 to $1.216 ; \mathrm{p}=0.515$ ). Although patients receiving cisplatin-based chemotherapy had a higher incidence of nausea and vomiting, thrombocytopenia was more frequent with carboplatin-based chemotherapy. No statistically significant difference in treatment-related mortality between cisplatin and carboplatin was reported [10].

Pemetrexed is a chemotherapeutic agent chemically similar to folic acid and a member of the class of chemotherapy drugs called folate antimetabolites. It works by inhibiting three enzymes used in purine and pyrimidine synthesis, thymidylate synthase, dihydrofolate reductase, and glycinamide ribonucleotide formyl transferase. By inhibiting the formation of precursor purine and pyrimidine nucleotides, pemetrexed prevents the formation of DNA and RNA, which are required for the growth and survival of both normal and cancer cells [11].

Its benefit was addressed in a trial conducted by Scagliotti and colleagues in which patients were randomized to receive either cisplatin $75 \mathrm{mg} / \mathrm{m}^{2}$ on day 1 and gemcitabine $1250 \mathrm{mg} / \mathrm{m}^{2}$ on days 1 and 8 or cisplatin $75 \mathrm{mg} / \mathrm{m}^{2}$ and pemetrexed $500 \mathrm{mg} / \mathrm{m}^{2}$ on day 1 , every 3 weeks for up to six cycles. Overall survival was statistically superior for cisplatin/pemetrexed versus cisplatin/gemcitabine in patients with adenocarcinoma (12.6 vs. 10.9 months, respectively) and large-cell carcinoma histology (10.4 vs. 6.7 months, respectively). In contrast, patients with squamous cell histology, showed a significant improvement in survival with cisplatin/gemcitabine versus cisplatin/pemetrexed; (10.8 vs. 9.4 months, respectively). For cisplatin/pemetrexed, rates of grade 3 or 4 neutropenia, anemia, and thrombocytopenia $(\mathrm{P}<0.001)$; febrile neutropenia $(\mathrm{P}=0.002)$; and alopecia $(\mathrm{P}<0.001)$ were significantly lower, whereas grade 3 or 4 nausea $(\mathrm{P}$ $=0.004)$ was more common [12].

Vascular endothelial growth factor (VEGF) is a potent endothelial-specific angiogenic factor that is expressed in a wide array of tumors. In NSCLC, high levels of VEGF expression was associated with a poor prognosis, suggesting that treatment targeting this pathway might be useful [13].

One approach to blocking the VEGF pathway is the administration of bevacizumab, a recombinant humanized monoclonal antibody that binds VEGF-A, thereby preventing its interaction with the VEGF receptor.

In an ECOG trial (E4599), previously untreated patients with advanced, non-squamous NSCLC were randomly assigned to paclitaxel plus carboplatin with or without bevacizumab $(15 \mathrm{mg} / \mathrm{kg})$ on day one of each cycle. Bevacizumab was continued as monotherapy on the same schedule after completion of six 
cycles of chemotherapy until progression. Patients receiving chemotherapy plus bevacizumab had statistically significant increases in the objective response rate (35 versus 15 percent with paclitaxel plus carboplatin alone), median overall survival (12.3 versus 10.3 months), one-year and two-year survival rates (51 versus 44 and 23 versus 15 percent, respectively), and progression-free survival (6.2 versus 4.5 months). Rates of clinically significant bleeding were $4.4 \%$ for chemotherapy-plus-bevacizumab group vs. $0.7 \%$ for chemotherapy alone group $(\mathrm{P}<0.001)$. The rates of $\geq$ grade 3 hypertension, bleeding, and proteinuria were modestly higher in the bevacizumab arms than in the control arm. There were 15 treatment-related deaths in the chemotherapy-plus-bevacizumab group, including 5 from pulmonary hemorrhage, versus 2 in the control arm [14].

In a meta-analysis based upon four trials conducted by Soria et al. that included 2194 patients, the addition of bevacizumab significantly increased both overall survival and progression-free survival compared with chemotherapy alone (HR 0.90, 95\% CI $0.81-0.99$ and 0.72, 95\% CI 0.66 - 0.79, respectively). The effect on overall survival was significantly greater in patients with adenocarcinoma compared with other histologies, however, bevacizumab significantly increased the risk of grade $\geq 3$ proteinuria, hypertension, hemorrhagic events, neutropenia, and febrile neutropenia [15].

In recent years there has been a major paradigm shift in the management of NSCLC with introduction of targeted therapy and immunotherapy for metastatic NSCLC, but most of these drugs require the presence of specific driver mutations. While up to $60 \%$ of patients with NSCLC have driver mutation, in the majority of cases there still are no effective drugs to target these and conventional chemotherapy remains an appropriate treatment option [16].

While randomized clinical trials have demonstrated the benefits of chemotherapy in carefully selected NSCLC patients, most NSCLC chemotherapy trials have stringent entry criteria that exclude patients with significant comorbidities or substantial functional impairment. Standard of care recommendations, therefore, are largely based on clinical trials limited to a select subpopulation of patients and how generalizable these results to other NSCLC patients still is unresolved [17] [18] [19].

\section{Methods}

A retrospective review of all NSCLC patients treated at University of Alabama at Birmingham, an NCI-designated comprehensive cancer center, from 2002 to 2012.

Stage IV NSCLC patients treated with standard of care first-line chemotherapy were identified. Four commonly used standard of care regimens were selected for review: carboplatin/paclitaxel (CT); carboplatin/gemcitabine (CG); carboplatin/pemetrexed (CP); carboplatin/paclitaxel/bevacizumab (CTB).

Details of patients' characteristics including comorbidities, chemotherapy regimens and survival were collected. 


\section{Results}

In our data, $21.1 \%$ of the patients were over 70 years old and $78.8 \%$ were less than 70 years old. Males comprised $60.8 \%$ of the patients and $39.2 \%$ were females and the majority of patients (84.5\%) were smokers. Almost half (49.7\%) the patients had adenocarcinomas, followed by non-small cell lung cancer not otherwise specified (31\%), followed by squamous cell carcinoma (19\%) (Table 1).

Most patients (66.5\%) had an ECOG 1 performance status, while $19.5 \%$ and $13.5 \%$ of patients respectively had performance statuses of ECOG 2 and ECOG 0. Less than $1 \%$ patients had an ECOG PS of 3 (Figure 1).

Regarding comorbidities, $38.1 \%$ of the patients had hypertension, $11.9 \%$ were diabetic, $23.7 \%$ had chronic obstructive pulmonary disease (COPD), $11.9 \%$ had coronary artery disease (CAD) and $2.1 \%$ had renal or liver disease (Table 2).

In patients treated with paclitaxel and carboplatin the median progression free survival was 4.9 months for patients responding to the regimen and overall survival was 13.1 months for responders vs. 9.2 months for non-responders.

Patients treated with Gemcitabine and carboplatin had a median progression free survival of 4.8 months for those responding to the regimen and overall survival was 13 months for responders vs. 8.9 months for non-responders.

Patients treated by pemetrexed and carboplatin had median progression free survival was 7.1 months for patients responding to this regimen and overall survival was 15.5 months for responders vs. 5.9 months for non-responder group of patients.

For patients treated by paclitaxel and carboplatin plus bevacizumab, the median progression free survival was 7.3 months for patients responding to the

Table 1. Demographic charters of the patients.

\begin{tabular}{|c|c|c|c|c|c|}
\hline & & Carboplatin/paclitaxel & Carboplatin/gemcitabine & Carboplatin/pemetrexed & $\begin{array}{c}\text { Carboplatin/ } \\
\text { paclitaxel/bevacizumab }\end{array}$ \\
\hline & & 106 & 35 & 25 & 28 \\
\hline \multirow{2}{*}{ Age } & $\geq 70$ & $16.0 \%$ & $31.4 \%$ & $24.0 \%$ & $25.0 \%$ \\
\hline & $<70$ & $84.0 \%$ & $68.6 \%$ & $76.0 \%$ & $75.0 \%$ \\
\hline \multirow{2}{*}{ Gender } & Female & $35.8 \%$ & $48.6 \%$ & $40.0 \%$ & $39.3 \%$ \\
\hline & Male & $64.2 \%$ & $51.4 \%$ & $60.0 \%$ & $60.7 \%$ \\
\hline \multirow{2}{*}{ Smoker } & Yes & $88.3 \%$ & $91.2 \%$ & $88.0 \%$ & $71.4 \%$ \\
\hline & No & $11.7 \%$ & $8.8 \%$ & $12.0 \%$ & $28.6 \%$ \\
\hline \multirow{3}{*}{ Histology } & Adenocarcinoma & $42.6 \%$ & $28.6 \%$ & $88.0 \%$ & $67.9 \%$ \\
\hline & SCC & $24.8 \%$ & $22.9 \%$ & $0.0 \%$ & $10.7 \%$ \\
\hline & NSCLC NOS & $32.7 \%$ & $48.6 \%$ & $12.0 \%$ & $21.4 \%$ \\
\hline \multirow{3}{*}{ Differentiation } & Well differentiated & $3.8 \%$ & $18.2 \%$ & $16.7 \%$ & $0.0 \%$ \\
\hline & $\begin{array}{c}\text { Moderately } \\
\text { differentiated }\end{array}$ & $26.4 \%$ & $27.3 \%$ & $25.0 \%$ & $38.5 \%$ \\
\hline & Poorly differentiated & $69.8 \%$ & $54.5 \%$ & $58.3 \%$ & $61.5 \%$ \\
\hline
\end{tabular}


Table 2. Distribution of comorbidities among treated population.

\begin{tabular}{ccccc}
\hline & Carboplatin/paclitaxel & Carboplatin/gemcitabine & Carboplatin/pemetrexed & Carboplatin/paclitaxel/bevacizumab \\
\hline HTN & $37.7 \%$ & $37.1 \%$ & $56.0 \%$ & $25.0 \%$ \\
DM & $8.5 \%$ & $17.1 \%$ & $28.0 \%$ & $3.6 \%$ \\
COPD & $23.6 \%$ & $22.9 \%$ & $32.0 \%$ & $17.9 \%$ \\
CAD & $11.3 \%$ & $17.1 \%$ & $16.0 \%$ & $3.6 \%$ \\
Renal Disease & $2.8 \%$ & $2.9 \%$ & $0.0 \%$ & $0.0 \%$ \\
Liver Disease & $0.9 \%$ & $0.0 \%$ & $12.0 \%$ & $0.0 \%$ \\
\hline
\end{tabular}

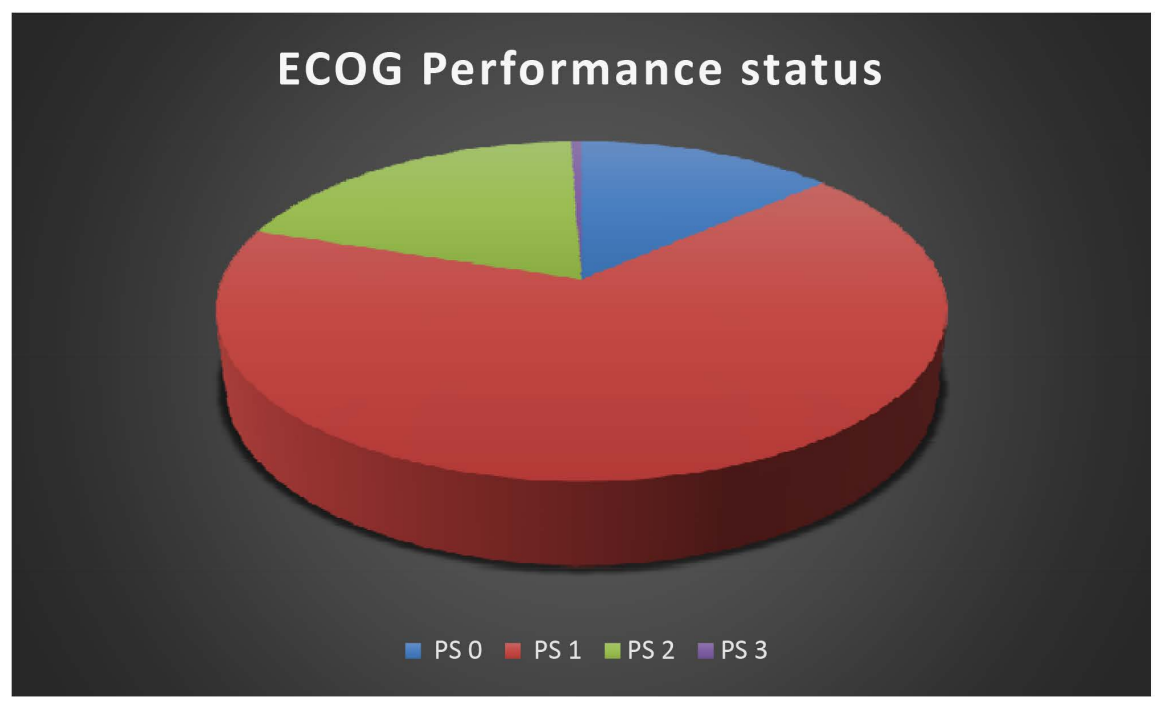

Figure 1. ECOG performance status.

regimen with an overall survival of 16.7 months versus 14.6 months for the non-responder group of patients.

The presence of single or multiple comorbidities was strongly associated with low overall survival compared to matched patients with no comorbidities ( $\mathrm{p}=$ 0.007) (Table 3 and Figure 2).

\section{Discussion}

In recent years there has been a major paradigm shift in the management of NSCLC with the introduction of targeted therapies for metastatic NSCLC. However most of these therapies require the presence of specific driving mutations and are not appropriate for most patients. For the majority of NSCLC without a driver mutation for which a drug is available, conventional chemotherapy remain a valid option [16].

Moreover, while randomized clinical trials have demonstrated the benefits of chemotherapy in carefully selected NSCLC patients, given the stringent entry criteria for these studies, they generally exclude patients with significant comorbidities or substantial functional impairment. The standard of care recommendations, therefore, are largely based on clinical trials limited to a select 
Table 3. Log rank test for comorbidities vs. no comorbidities $(\mathrm{p}=0.007)$.

\begin{tabular}{ccccc}
\hline & \multicolumn{4}{c}{ Median } \\
\cline { 2 - 5 } Comorbidities & Estimate & Std. Error & \multicolumn{2}{c}{$95 \%$ Confidence Interval } \\
\cline { 4 - 5 } & & & Lower Bound & Upper Bound \\
\hline Comorbidities & 11.108 & 0.682 & 9.771 & 12.446 \\
No comorbidities & 12.944 & 1.422 & 10.158 & 15.731 \\
Overall & 11.797 & 0.463 & 10.890 & 12.704 \\
\hline
\end{tabular}

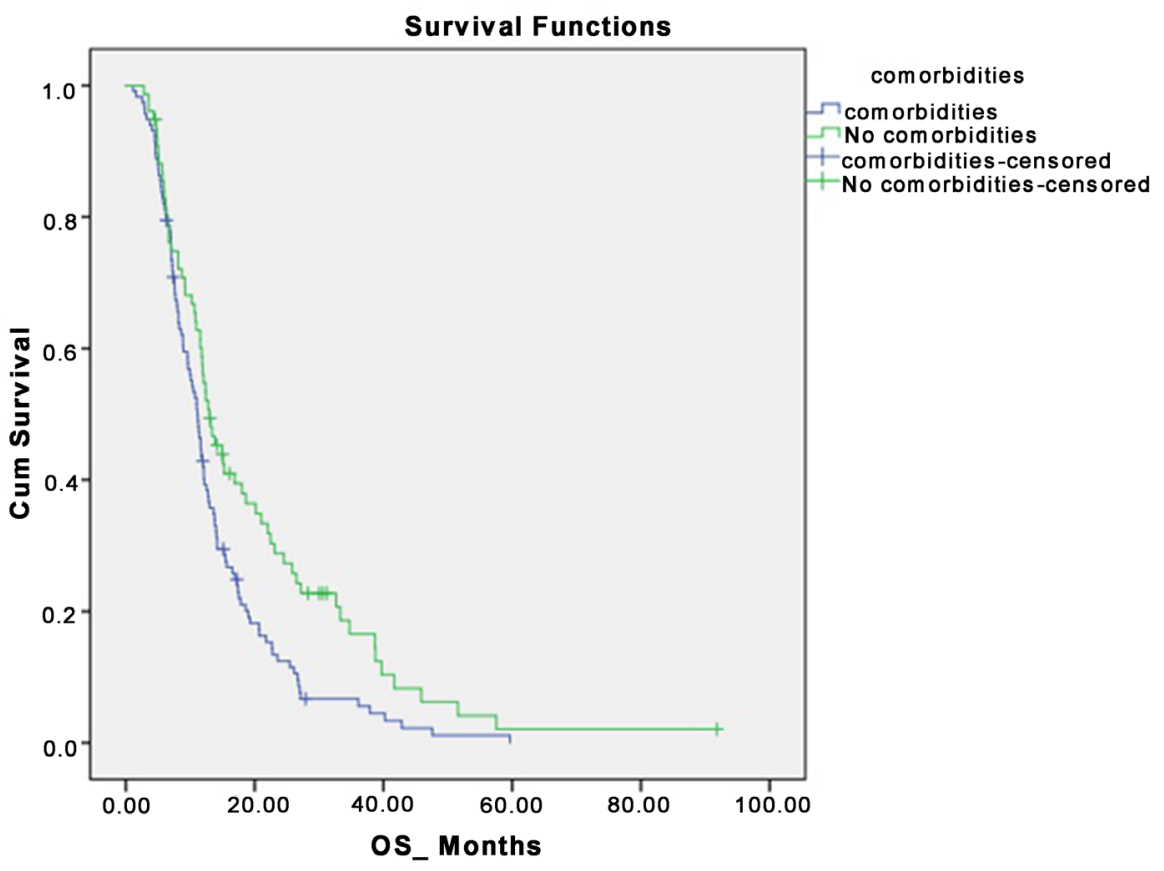

Figure 2. Kaplan Marie survival curve for comorbidities vs. no comorbidities ( $\mathrm{p}=0.007)$.

non-representative subpopulation of patients. How generalizable these results are to other NSCLC patients remains unresolved [17] [18] [19].

In our study four commonly used standard of care regimens were selected for review: carboplatin/paclitaxel (CT); carboplatin/gemcitabine (CG); carboplatin/pemetrexed (CP); carboplatin/paclitaxel/bevacizumab (CTB). Regarding comorbidities, $38.1 \%$ of the patients had hypertension, $11.9 \%$ of the patients were diabetic, $23.7 \%$ had chronic obstructive pulmonary Diseases (COPD), $11.9 \%$ had coronary artery diseases (CAD) and $2.1 \%$ had renal or liver diseases.

The effects of these comorbidities on outcome are well known. In one study conducted by Tammemagi and colleagues, COPD, liver or renal diseases were associated with inferior survival compare to a matched group [20]. In another study data reported by Kiri et al, who reviewed the UK GP Research Database, the three-year survival for lung cancer patients with a history of COPD was almost half that of the general population of lung cancer patients $(15 \%$ versus $26 \% ; \mathrm{p}<0.01)$ [21].

In the present study, the presence of single or multiple comorbidities among 
patients who received standard chemotherapy regimens was associated with low overall survival compare to matched patients with no comorbidities $(p=0.007)$.

\section{Conclusion}

The presences of single or multiple comorbidities are associated with inferior overall survival compare to matched groups without such conditions. Clinical practitioners should consider this in interpreting the results of clinical trials when making treatment recommendations for their patients. Similarly, consideration should be given in the design of clinical trials to accruing patients who more closely reflect the general of the NSCLC patient population.

\section{References}

[1] Ferlay, J., Ervik, M., Dikshit, R., Eser, S., Mathers, C., Rebelo, M., Parkin, D.M., Forman, D. and Bray, F. (2013) GLOBOCAN 2012 v1.0, Cancer Incidence and Mortality Worldwide. IARC Cancer Base No. 11.

[2] Jemal, A., et al. (2011) Global Cancer Statistics. CA: A Cancer Journal of Clinicians, 61, 69-90. https://doi.org/10.3322/caac.20107

[3] Beasley, M.B., Brambilla, E. and Travis, W.D. (2005) The 2004 World Health Organization Classification of Lung Tumors. Seminars in Roentgenology, 40, 90-97. https://doi.org/10.1053/j.ro.2005.01.001

[4] Travis, W.D., et al. (2015) The 2015 World Health Organization Classification of Lung Tumors: Impact of Genetic, Clinical and Radiologic Advances Since the 2004 Classification. Journal of Thoracic Oncology, 10, 1243-1260. https://doi.org/10.1097/JTO.0000000000000630

[5] DH, J. (1993) Chemotherapy for Metastatic Non-Small-Cell Lung Cancer Can That Dog Hunt. Journal of the National Cancer Institute, 85, 766-767. https://doi.org/10.1093/jnci/85.10.766

[6] Group, N.-S.C.L.C.C. (1995) Chemotherapy in Non-Small Celi Lung Cancer: A Meta-Analysis Using Updated Data on Individual Patients from 52 Randomised Clinical Trials. BMJ, 311, 899-909. https://doi.org/10.1136/bmj.311.7010.899

[7] Schiller, J.H., Harrington, D., Belani, C.P., Langer, C., Sandler, A., Krook, J., Zhu, J. and Johnson, D.H. (2002) Comparison of Four Chemotherapy Regimens for Advanced Non-Small-Cell Lung Cancer. New England Journal of Medicine, 346, 92-100. https://doi.org/10.1056/NEJMoa011954

[8] Smit, E.F., et al. (2003) Three-Arm Randomized Study of Two Cisplatin-Based Regimens and Paclitaxel Plus Gemcitabine in Advanced Non-Small-Cell Lung Cancer: A Phase III Trial of the European Organization for Research and Treatment of Cancer Lung Cancer Group-EORTC 08975. Journal of Clinical Oncology, 21, 3909-3917. https://doi.org/10.1200/JCO.2003.03.195

[9] Ohe, Y., et al. (2007) Randomized Phase III Study of Cisplatin Plus Irinotecan versus Carboplatin Plus Paclitaxel, Cisplatin Plus Gemcitabine, and Cisplatin Plus Vinorelbine for Advanced Non-Small-Cell Lung Cancer: Four-Arm Cooperative Study in Japan. Annals of Oncology, 18, 317-323.

https://doi.org/10.1093/annonc/mdl377

[10] Hotta, K., et al. (2004) Meta-Analysis of Randomized Clinical Trials Comparing Cisplatin to Carboplatin in Patients with Advanced Non-Small-Cell Lung Cancer. Journal of Clinical Oncology, 22, 3852-3859. 
https://doi.org/10.1200/JCO.2004.02.109

[11] McLeod, H.L., et al. (2000) Pharmacokinetic and Pharmacodynamic Evaluation of the Glycinamide Ribonucleotide Formyltransferase Inhibitor AG2034. Clinical Cancer Research, 6, 2677-2684.

[12] Scagliotti, G.V., Parikh, P., von Pawel, J., Biesma, B., Vansteenkiste, J., Manegold, C., Serwatowski, P., Gatzemeier, U., Digumarti, R., Zukin, M., Lee, J.S., Mellemgaard, A., Park, K., Patil, S., Rolski, J., Goksel, T., de Marinis, F., Simms, L., Sugarman, K.P. and Gandara, D. (2008) Phase III Study Comparing Cisplatin Plus Gemcitabine With Cisplatin Plus Pemetrexed in Chemotherapy-Naive Patients with Advanced-Stage Non-Small-Cell Lung Cancer. Journal of Clinical Oncology, 26, 9.

[13] Ellis, L.M. and Hicklin, D.J. (2008) VEGF-Targeted Therapy: Mechanisms of Anti-Tumour Activity. Nature Reviews Cancer, 8, 579-591. https://doi.org/10.1038/nrc2403

[14] Sandler, A., et al. (2006) Paclitaxel-Carboplatin Alone or with Bevacizumab for Non-Small-Cell Lung Cancer. New England Journal of Medicine, 355, 2542-2550. https://doi.org/10.1056/NEJMoa061884

[15] Soria, J.C., et al. (2013) Systematic Review and Meta-Analysis of Randomised, Phase II/III Trials Adding Bevacizumab to Platinum-Based Chemotherapy as First-Line Treatment in Patients with Advanced Non-Small-Cell Lung Cancer. Annals of Oncology, 24, 20-30. https://doi.org/10.1093/annonc/mds590

[16] Chan, B.A. and Hughes, B.G.M. (2015) Targeted Therapy for Non-Small Cell Lung Cancer: Current Standards and the Promise of the Future. Translational Lung Cancer Research, 4, 36-54.

[17] Members, N.P. (2017) NCCN Clincal Practice Guidelines, Non-Small Cell Lung Cancer.

[18] Hanna, N., et al. (2017) Systemic Therapy for Stage IV Non-Small-Cell Lung Cancer: American Society of Clinical Oncology Clinical Practice Guideline Update. Journal of Clinical Oncology, 35, 3484-3515. https://doi.org/10.1200/JCO.2017.74.6065

[19] Novello, S., et al. (2016) Metastatic Non-Small-Cell Lung Cancer: ESMO Clinical Practice Guidelines for Diagnosis, Treatment and Follow-Up. Annals of Oncology, 27, v1-v27. https://doi.org/10.1093/annonc/mdw326

[20] Tammemagi, C.M., et al. (2003) Impact of Comorbidity on Lung Cancer Survival. International Journal of Cancer, 103, 792-802. https://doi.org/10.1002/ijc.10882

[21] Kiri, V.A., et al. (2009) Recent Trends in Lung Cancer and Its Association with COPD: An Analysis Using the UK GP Research Database. Primary Care Respiratory Journal, 19, 57-61. https://doi.org/10.4104/pcrj.2009.00048 